\title{
Modellhafte Register für die Versorgungsforschung
}

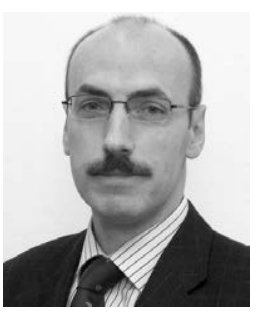

Jürgen Stausberg
Bibliografie

Gesundheitswesen 2021; 83 (Suppl. 1): S1-S3

DOI $10.1055 / \mathrm{a}-1654-4105$

ISSN $\quad 0949-7013$

(c) 2021. Thieme. All rights reserved.

Georg Thieme Verlag, Rüdigerstraße 14,

70469 Stuttgart, Germany

Korrespondenzadresse

Prof. Dr. med. Jürgen Stausberg

Institut für Medizinische Informatik

Biometrie und Epidemiologie (IMIBE)

Universitätsklinikum Essen (AöR)

Hufelandstraße 55

45147 Essen

Deutschland

juergen.stausberg@uk-essen.de
Register sind eine etablierte und bewährte Methode der Versorgungsforschung [1]. Umso weitsichtiger war es vom Bundesministerium für Bildung und Forschung (BMBF) im Jahre 2016, mit einer Ausschreibung zum Aufbau modellhafter patientenbezogener Register für die Versorgungsforschung die Zukunftsfähigkeit und Innovationskraft dieser Methode zu unterstreichen (s. https://www. gesundheitsforschung-bmbf.de/de/modellhafte-register-realisierungsphase-9011.php). Hierbei hatte das BMBF mit einer Zweiteilung der Förderung in eine Konzepterstellung und eine Realisierung die Bedeutung der systematischen Entwicklung eines Registers hervorgehoben und damit die Impulse aus dem Deutschen Netzwerk Versorgungsforschung (DNVF) für ein Registerprotokoll [2] aufgenommen. Aus 16 Projekten wurden sechs Register für eine Umsetzung ausgewählt, die sich zusammen mit einem Begleitprojekt in diesem Sonderheft der Zeitschrift „Das Gesundheitswesen“ präsentieren.

Gegenstand von Registern ist die systematische Betrachtung des Alltags der Gesundheitsversorgung. Dies unterscheidet Register auf der einen Seite von klinischen Studien, die eine höchstmögliche Einheitlichkeit von Versorgungsbedingungen anstreben, um Unterschiede interessierender diagnostischer oder therapeutischer Verfahren herauszuarbeiten. Auf der anderen Seite setzen Register der Beliebigkeit von Anwendungsbeobachtungen vorab definierte Ziele, Aufgaben und Fragestellungen entgegen. Die sechs ausgewählten Register betrachten dazu ganz unterschiedliche Versorgungssituationen, die Nierenlebendspende, die nicht-infektiöse Uveitis, die Querschnittlähmung, rezidivierende Steinerkrankungen des oberen Harntraktes, erblichen Brust- und Eierstockkrebs sowie Fieber [3]. Damit befassen sich die Register der Fördermaßnahme mit akuten wie mit chronischen Erkrankungen, mit Erkrankungen bei Kindern, Jugendlichen und Erwachsenen, mit Gesunden wie Kranken sowie mit der Onkologie. Sie bilden damit aus me- dizinischer Sicht einen bunten Strauß möglicher Einsatzfelder für die Gewinnung von Erkenntnissen aus Registern ab. Sie sind für den jeweils betrachteten Gegenstandbereich eine „Antwort auf erweiterte Informationsbedürfnisse zur Versorgungssituation im Gesundheitswesen“ “ [4]. Dieses Informationsbedürfnis ist seit Anfang 2020 durch die Corona-Pandemie massiv gestiegen und in den Fokus der Öffentlichkeit gelangt. Die Aktualität von Registern wird daher durch die Ergänzung eines Beitrags zum „Lean European Open Survey on SARS-CoV-2 Infected Patients (LEOSS) “ [5] in diesem Sonderheft unterstrichen.

Zukunftsfähigkeit und Innovationskraft von Registern sind auch international erkannt. So verknüpft das Handbuch der US-amerikanischen Agency for Healthcare Research and Quality (AHRQ) in seiner aktuellen, vierten Auflage [6] Patientenregister mit der Digitalisierung im Gesundheitswesen. Patienten sind nicht länger nur passive Beobachtungseinheiten, sondern werden auch als aktive Partner bei Planung, Entwurf, Umsetzung und Betrieb eines Registers eingebunden. Diese Perspektiven finden sich in der Modellhaftigkeit der geförderten Projekte wieder. So erfassen beim FieberApp-Register Familienangehörige Angaben in einer App, die dann in einem zentralen Datenbestand zusammengeführt werden [7]. Etablierte Methoden zur Qualitätssicherung von Daten wie Monitoring oder Query-Management müssen in diesem Setting neu gedacht werden. Bei der Auswertung des FieberApp-Registers ist es dann der Perspektive überlassen, Familien, Familienangehörige, Kinder und Jugendliche oder Fieberepisoden als Beobachtungseinheit zu verstehen. Je nach Perspektive werden die Ergebnisse unterschiedlich ausfallen.

Auch beim Register RECUR zu rezidivierenden Steinerkrankungen des oberen Harntraktes wird eine App für Patienten zur Gewinnung von Informationen über den Verlauf der Erkrankung eingesetzt [8]. Kaum ein Register kann noch auf Angaben zu patienten- 
berichteten Endpunkten (Patient Reported Outcome Measures, PROMs) wie Lebensqualität verzichten, vielfach direkt durch die Patienten erfasst. Als zweite Quelle nutzt RECUR Routinedaten der Versorgung. So werden einmal erfasste Daten mehrfach genutzt und zusätzlicher Eingabeaufwand vermieden. Datenbestände, hier Routinedaten der Versorgung und Eingaben der Patienten, werden miteinander verknüpft. Eine Verknüpfung von Datenbeständen bietet sich auch zwischen Registern an, z. B. entlang eines Versorgungsprozesses. Das Register SOLKID-GNR zur Sicherheit des Lebendnierenspenders [9] könnte sich so mit einem auf die Empfänger bezogenen Transplantationsregister verbinden. Der Organspender bei SOLKID-GNR ist nicht krank, aber Patient durch seinen Kontakt mit dem Transplantationszentrum. Es wird interessant sein, zu verfolgen, ob die Betrachtung von gesundheitsrelevanten Fragestellungen bei Gesunden ein Register vor andere Herausforderungen stellt, als dies bei Kranken der Fall ist. Auch Personen mit einer genetischen Vorbelastung für Brust- und Eierstockkrebs sind nicht krank; bei Verwandten kann selbst die Risikokonstellation fehlen. So befasst sich HerediCaRe mit der risiko-adaptierten Prävention für Krebserkrankungen [10]. Während Register typischerweise bei einer Erkrankung aufsetzen und deren Verlauf beschreiben, betrachtet HerediCaRe die Entstehung von Erkrankungen bei vorliegender Risikokonstellation. Selbstverständlich spielen PROMs auch bei HerediCaRe eine wichtige Rolle, wie bei allen Registern der Fördermaßnahme.

Das Register ParaReg zum lebenslangen Monitoring von Querschnittgelähmten befasst sich mit einem Zustand nach Verletzung oder als Folge einer nicht-traumatischen Erkrankung, der Querschnittlähmung [11]. Im Rahmen von ParaReg können verschiedene Behandlungspfade verfolgt und hinsichtlich ihrer Behandlungsergebnisse miteinander verglichen werden. Die lebenslange Nachverfolgung von Patienten ist typisch für Register in der Versorgungsforschung und ein Grund, warum der erfolgreiche Betrieb eines Registers einen langen Atem voraussetzt. Das Register TOFU zu Behandlungsaustrittsoptionen bei nicht-infektiöser Uveitis ist ein Beispiel für ein Arzneimittelregister, bei dem Wirkung, unerwünschte Folgen und Komplikationen einer Pharmakotherapie im Mittelpunkt stehen [12]. Auch hierzu gehört die Erfassung von PROMs, in diesem Falle nicht durch eine App für Smartphones, sondern durch einen webbasierten Zugriff auf Fragebögen eines Patientenmoduls. TOFU kann zudem zu Registern für seltene Erkrankungen gerechnet werden.

Die sechs Register der Fördermaßnahme, das FieberApp-Register, HerediCaRe, ParaReg, RECUR, SOLKID-GNR und TOFU, spiegeln zusammen mit LEOSS die Vielfalt von Registern in Deutschland wider, die aus der Wissenschaft initiiert und von Forschern organisiert werden. Diese Vielfalt gilt es zu erhalten. Die Register belegen mit ihren modellhaften Ansätzen, dass die Methode zukunftsfähig und offen für Innovationen ist. Digitalisierung des Gesundheitswesens und aktive Einbindung von Patienten sind dabei zwei Treiber, die sich durchgängig in den Ansätzen der Register wiederfinden. Eine Regulierung von Registern für die Versorgungsforschung, wie sie im Bereich der Nutzenbewertung für die anwendungsbegleitende Datenerhebung nach $\S 35$ a Absatz 3b Sozialgesetzbuch (SGB) V erfolgt [13], ist daher weder sinnvoll noch notwendig. Ungelöst ist die Sicherstellung einer nachhaltigen Finanzierung von Registern, deren Anspruch auf eine lebenslange Nachverfolgung von Patienten Laufzeiten über Jahrzehnte bedingt. Angesichts der zunehmenden Erfassung von PROMs durch Patienten böte es sich an, die hierfür eingesetzten Werkzeuge als erstattungsfähige digitale Gesundheitsanwendungen (DiGa) nach § 33a SGB V auszuweisen. Hierfür wäre die Erfassung von PROMs für Register als Bestimmung einer DiGa anzuerkennen. Auf diese Weise könnten beteiligte Leistungserbringer zumindest für ihre Unterstützung bei der Rekrutierung von Patienten eine finanzielle Kompensation von Seiten der Krankenkassen erhalten.

Aus einem Verzicht auf Regulierung und der Forderung nach einer öffentlichen Finanzierung leiten sich aber auch Ansprüche an Register in der Versorgungsforschung ab, um einen bestmöglichen Nutzen für die Gesellschaft zu erreichen. So sollten Existenz, Gegenstand und Rahmenbedingungen eines Registers in einem „Register der Register" nachvollziehbar dargestellt sein. Die Inhalte eines Registers wären in einem Katalog von Metadaten einsehbar. Veröffentlichungen von Ergebnissen aus Registern werden mit quantitativen Angaben zur Datenqualität, idealerweise unter Nutzung etablierter Indikatoren, hinterlegt. Auch diesen Ansprüchen an Transparenz stellt sich die Fördermaßnahme des BMBF zum Aufbau modellhafter patientenbezogener Register für die Versorgungsforschung. U. a. ist es Aufgabe des Begleitprojekts, sich für diese Transparenz über die geförderten Register einzusetzen [3].

\section{Danksagung}

Das Begleitprojekt REGISVF-AP wird vom BMBF unter dem Kennzeichen 01GY1917B gefördert. Der Autor ist den Gutachterinnen und Gutachtern für ihre Reviews zu den Beiträgen des Sonderhefts zu Dank verpflichtet.

\section{Interessenkonflikt}

Die Autorinnen/Autoren geben an, dass kein Interessenkonflikt besteht.

\section{Literatur}

[1] Bestehorn K. Medizinische Register. Med Klin 2005; 100: 722-728

[2] Müller D, Augustin M, Banik N et al. Memorandum Register für die Versorgungsforschung. Das Gesundheitswesen 2010; 72: 824-839

[3] Harkener S, Stausberg J. Projektübergreifende Unterstützung von Registern in Entwicklung, Umsetzung und Betrieb. Das Gesundheitswesen 2021; 83 (Suppl. 1): S54-S59

[4] Mathis S, Wild C. Register für klinische und gesundheitsökonomische Fragestellungen. HTA-Projektbericht Nr. 11. Wien: Ludwig Boltzmann Gesellschaft GmbH; 2008

[5] Pilgrim L, Schons M, Jakob C et al. Die COVID-19 Pandemie als Herausforderung und Chance für Register in der Versorgungsforschung: Erfahrungen aus Lean European Open Survey on SARS-CoV-2 Infected Patients (LEOSS). Das Gesundheitswesen 2021; 83 (Suppl. 1): S45-S53

[6] Gliklich RE, Leavy MB, Dreyer NA. eds. Registries for Evaluating Patient Outcomes: A User's Guide. $4^{\text {th }}$ ed. AHRQ Publication No. 19(20)-EHC020. Rockville, MD: Agency for Healthcare Research and Quality; 2020 
[7] Jenetzky E, Schwarz S, Fingerhut I et al. Das FieberApp Register - ein Weg, um Eltern durch eigene Dokumentation zu einer abgestuften Entscheidung zu führen. Erste Erfahrungen mit der FeverApp für Familien. Das Gesundheitswesen 2021; 83 (Suppl. 1): S4-S11

[8] Walther T, Farin E, Boeker M et al. RECUR - Aufbau eines automatisierten digitalen Registers für Patient*innen mit rezidivierenden Steinen des oberen Harntraktes. Das Gesundheitswesen 2021; 83 (Suppl. 1): S27-S32

[9] Suwelack B, Dugas M, Koch M et al. Die Sicherheit des Lebendnierenspenders - Das Deutsche Lebendspende Register SOLKID-GNR - Entstehung und Struktur eines nationalen Registers in der Versorgungsforschung. Das Gesundheitswesen 2021; 83 (Suppl. 1): S33-S38

[10] Engel C, Wieland K, Zachariae S et al. HerediCaRe: Dokumentationsund IT-Lösung eines spezialisierten Registers für erblichen Brust- und Eierstockkrebs. Das Gesundheitswesen 2021; 83 (Suppl. 1): S12-S17
[11] Rupp R, Jersch P, Schuld C et al. Das deutschlandweite, webbasierte ParaReg-Register zur lebenslangen Dokumentation von Querschnittgelähmten - Datenmodell, rechtlich-ethische Voraussetzungen und technische Implementierung. Das Gesundheitswesen 2021; 83 (Suppl. 1): S18-S26

[12] Li JQ, Dell J, Höller T et al. Das Treatment Exit Options for Uveitis (TOFU) Register: Einbindung von Patienten in die Evidenzgenerierung. Das Gesundheitswesen 2021; 83 (Suppl. 1): S39-S44

[13] Niemeyer A, Kluge S, Gurisch C et al. Positionspapier des Deutschen Netzwerk Versorgungsforschung (DNVF) zur anwendungsbegleitenden Datenerhebung nach Sozialgesetzbuch V. Das Gesundheitswesen 2021; 83: 309-313 